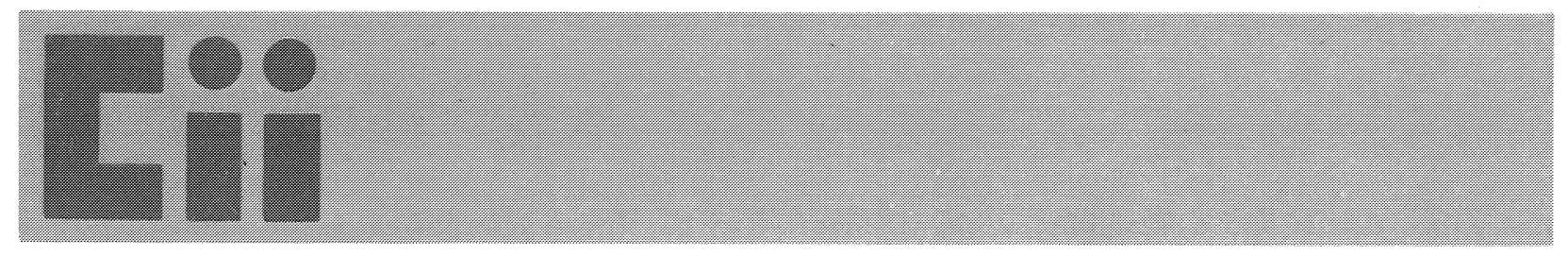

\title{
SOLUCIONES ACTUALES AL PROBLEMA DE LA VIVIENDA EN IBEROAMERICA
}

\author{
(CURRENT SOLUTIONS TO THE HOUSING PROBLEM IN LATIN AMERICA)
}

\author{
Horacio Berretta Garcia, Arquitecto \\ con la colaboración de \\ Marta Mendizábal de Berretta \\ Centro Experimental de la Vivienda Económica \\ - CEVE- de Córdoba, (AVE-CONICET) \\ ARGENTINA
}

\section{RESUMEN}

La tecnología poderosa y evolucionada de la sociedad productiva y consumista acrecienta día a día las distancias entre ricos y pobres.

En el campo de la vivienda, el «desarrollo para los desarrollados" ha generado y promocionado un estereotipo prestigioso de tecnología avanzada y vivienda convencional («llave en mano»), para quien la pueda pagar, que traba, sin lugar a dudas, la urgente y masiva realización de lo factible, postergando a un hipotético futuro de grandeza y abundancia las necesidades esenciales del grueso de la población. La tecnotecnología adecuada requiere diseñar el proceso de construir, de modo tal, que posibilite la participación popular en el mismo.

También implica dar cabida a que dicho proceso pueda, en Planes de Vivienda y Promoción, centrarse en el Objetivo integrado: Organización grupal en la construcción de la vivienda y el barrio, como camino de afirmación familiar y auto-gestion comunitaria.

La búsqueda de tecnología interdisciplinaria, adecuada para estos planes, presupone recorrer un largo camino de investigación, experimentación y evaluación en ciclos ininterrumpidos.

Esta es la tarea central a la que se halla abocada la Asociación Vivienda Económica (AVE), en Córdoba, Argentina. Se describe, a manera de ejemplo, uno de los planes piloto de Vivienda y Promoción realizados por esta Institución.

\section{SUMMARY}

The powerful and greatly developed technology of the productive consumer society is increasing the gap between rich and poor daily.

In the sphere of housing, the idea of «development for the developed» has generated and promoted a prestigious stereotype of conventional housing with advanced technology: («key in hand»), for those who can afford it, which, without the shadow of a doubt, hinders the urgent execution en masse of what is practicable, leaving the essential needs of the bulk of the population to a hypothetical future of abundance and splendour. Adequate technology requires designing the building process in such a way that it is possible for people to take part in it.

It also implies making it possible for this process to be centred on the integrated Objective, in the Housing and Promotion Plans: Group Organization in the construction of housing and the neighbourhood, leading to an affirmation of the family and self-administration for the community.

The search for adequate interdisciplinary technology for these plans presupposes travelling down a long road of research, experimentation and evaluation in uninterrupted cycles.

This is the central theme which the Asociacion Vivienda Economica ( $A V E$ ), (Economic Housing Association) in Córdoba, Argentina, has arrived at. One of the Housing and Promotion pilot plans, carried out by this Institution, is described as an example.

\section{INTRODUCCION}

Las diferencias entre paises ricos y pobres aumentan dia a dia. La tecnología poderosa y evolucionada de la sociedad productiva y consumista, acrecienta distancias, de tal manera que a la revolución del carbón y del petróleo, le seguirá la de la electrónica, mientras el grueso de nuestros pueblos del Tercer Mundo continúan consumiendo energias, tal como lo hacian hace $100 \delta 1.000$ años, y sin muchas posibilidades de cambio.
El consumo de los ricos se hace mayor y cierra las puertas a la satisfacción de las necesiciades básicas de los pobres, que son la mayoria.

Asi en el campo de la vivienda, las aparentes buenas intenciones de solución masiva, se estrellan con las reales posibilidades, y la vivienda ideal "llave en mano" no llega a los sectores mayoritarios. No es una afirmación gratuita, es una constatación más, que, como en el campo del consumo energético, pone odiosas e imposibles distan- 
cias entre las metrópolis imperiales y los pueblos pobres.

Las soluciones actuales al problema de la vivienda masiva pasa, para algunos, por la definición de politicas en que la tecnologia de avanzada ha de dar la última palabra; para otros probablemente pasa por la reiteración de las viejas políticas en que un sector realiza su vivienda moderna y costosa y el grueso se las arregla como puede.

Para otros, como es nuestro caso, las soluciones han de ser múltiples y complementarias, pasando las diversas alternativas compatibles, no tanto por lo que sueñan los políticos o técnicos de gabinete, cuanto por lo que realmente es posible utilizando escasos recursos, en profunda comunión con nuevas formas de promoción generalizada de las mayorias olvidadas o marginadas.

Hoy como ayer ellas autoconstruyen su guarida, su rancho, su casita orillera, y en esta capacidad de creativa subsistencia, los políticos, los técni$\cos$ y los investigadores deben saber encontrar todo el rico potencial que ello comporta.

Alargar los brazos de los pobres trabajando con ellos para poner en sus manos: medios, técnicas y herramientas aptas para realizaciones más racionales, posibles y adecuadas a sus necesidades sentidas, es la tarea requerida para encontrar las debidas "soluciones actuales al problema de la vivienda en Iberoamérica" y en general en los paises en desarrollo, en el marco de la construcción de una nueva civilización universal y solidaria que pueda compatibilizar justicia y libertad.

\section{LAS ALTERNATIVAS}

Pese a la rápida urbanización, la mitad de la población mundial vive aún en aldeas de 100 a 1.000 habitantes, 0 esparcidas en bosques, montañas 0 desiertos.

Mientras la riqueza se encuentra en las ciudades, el campo que sigue siendo la fuente del alimento de los paises ricos y de las ciudades del Tercer Mundo, soporta abandono y miseria. Sus uavanzadas" sitian con sus Villas Miseria las ciudades, pero el grueso de sus pobladores permanece aún atado a la pobreza y el subdesarrollo.

¿Debemos esperar que todo el campo invada la ciudad para destruirla desde la periferia y atarla de pies y manos inmovilizándola? ¿O debemos dejarlo agonizar con sus hombre aguardando el sustento de manos de las máquinas de las grandes Babilonias?

La tecnologia y el desarrollo deben servir al hombre, -multitud sumergida en la pobreza- y buscar la fuente de la riqueza y la vida que no está sólo en las urbes, actualmente infectadas, invadi- das de afuera y minadas desde adentro por la opulencia y la deshumanización, sino también en el campo, hoy en agonía.

Estos son los reales problemas del mundo y no visitar la luna, perfumar el aire acondicionado, acelerar la velocidad del ascensor, manipular cromosomas para fabricar "gemelos productores", crear más armas nucleares, hacer televisores de bolsillo y diseñar viviendas electrónicas...

En los paises del Tercer Mundo a las tecnologias "empíricas" o "espontáneas" de la vivienda, heredadas de culturas muchas veces milenarias, aunque ya desestabilizadas, se han agregado tecnologias desarrolladas en los paises colonizadores o producidas en los centros del Hemisferio Norte.

Los campesinos que siguen construyendo como 100 ó 500 años atrás, cuando son impelidos a marchar a la periferia de las ciudades, realizan su "cobijo" con técnicas que son resultantes de sus experiencias ancestrales, a las que incorporan las tecnologias extrañas, como pueden.

Las primeras vinculadas a un pasado que no ha evolucionado y también a la miseria y el subdesarrollo; las segundas estrechamente relacionadas a intereses y apetencias crecientes de las minorias ricas y al gusto de técnicos y profesionales formados "a la europea".

Una mirada globalizadora, sobre la situación de nuestras masas pobres, muestra por un lado el rechazo y desinterés de la sociedad moderna y los gobiernos por la suerte de los pobres, y por otro, que el grueso de las tecnologias desarrolladas en todos los campos, son inadecuadas para las mayorias.

Hoy, ¿qué es más razonable? ¿Ampliar la fabricación de herramientas de alta tecnología, mirando a la perfección del producto restrictivo? ¿O la búsqueda del autoabastecimiento de las mayorias, en procedimientos más simples y generalizables...?

Los gobiernos de los paises en desarrollo con la visión tomada de los Imperios, casi siempre optan por lo primero a espaldas de sus propios pueblos, aunque con la ilusión de un generalizado desarrollo y riqueza que nunca llega...

Se insiste en que lo que es bueno para los ricos a la larga lo es también para los pobres, tratando de no ver que los frutos de este "buen deseo", se concretan en aumentar los privilegios de los primeros y la miseria de las mayorias. Asi para prevenir los peligros del enfrentamiento y la lucha de clases se apuntalan los privilegios de las clases más fuertes y privilegiadas.

Sin embargo, el "desarrollo para los desarrollados", ha generado y promocionado un estereotipo prestigioso de vivienda convencional y tecnologia 
también convencional, que traba sin lugar a dudas, la urgente realización masiva de lo posible.

La imagen de una tecnología moderna que cubra idealmente la totalidad de las necesidades de la población, se ha traducido en la práctica, en dos modalidades bien diferentes: para quienes pueden pagar, tenemos "la vivienda llave en mano". Quienes no le pueden pagar (aunque sea el sector mayoritario), deben apelar sin apoyo oficial, al "cobijon, al tugurio o a la vivienda autogestionada o autoconstruida... ¡Allá se las arreglen!

Esta concepción determina la discontinuidad, aunque también las semejanzas, de las políticas habitacionales del Tercer Mundo, que en nombre de la modernización y la eficiencia técnica, desestiman generalmente la concreción de todo apoyo para la vivienda informal y formas constructivas no convencionales, postergando a un hipotético futuro de grandeza y abundancia, las necesidades esenciales del grueso de la población.

Asi pues, las políticas habitacionales (con escasas excepciones), apoyan exclusivamente a la vivienda ideal: "llave en mano" y a la vivienda mercancia, como índice claro de progreso y desarrollo.

Dado que las caracteristicas de calidad y apariencia adoptadas por los gobiernos, son la preocupación fundamental de los organismos públicos de crédito y control, es explicable que la atención de los sectores marginados y de recursos insuficientes, no vaya más allá de buenas intenciones y promesas de realización imposible.

Los fondos oficiales terminan beneficiando a las clases medias y altas, y a las grandes empresas constructoras, creando un círculo vicioso en una política cada vez más selectiva y excluyente, aunque de renombrado prestigio entre funcionarios y profesionales admiradores de la "perfección del producto..." y del desarrollo de los que ya son ricos.

Sin embargo, lentamente la idea de políticas y tecnologias adecuadas a las necesidades impostergables de las masas, va surgiendo, justo a la visualización de un nuevo proyecto de sociedad más humana y justa, como una nueva visión del mundo al margen de las luchas imperiales del Este y el Oeste.

Esta visión, para que no derive en acciones aisladas o palabras de moda, debe estar vinculada a un modelo de desarrollo democrático, participativo, justo y libre.

Debe desarrollar diversas alternativas para un verdadero servicio a la sociedad global.

Por esto, junto al apoyo a las empresas tradicionales que sirven a un sector de la población, el Estado debe orientar una parte significativa de sus recursos del área vivienda e investigación, a la generación de tecnologías simples para la autogestión, el trabajo de participación comunitaria, programas de "ayuda mutua", "esfuerzo propio", "mixtos", etc.

Esto supone a su vez, una descentralización parcial en la toma de las decisiones relativas a la construcción que generalmente se halla monopolizada por intereses potentes que dan respuesta habitacional a quienes pueden pagar el servicio, con fondos oficiales o propios.

Supone entonces un camino distinto que el determinado por políticas y tecnologias que tienden cada vez más a afirmar las ideas del monopolio y de una industrialización que comporta agrandar el tamaño de las empresas con la excusa de lograr un servicio más eficaz, reduciendo mano de obra y propiciando la fabricación y montaje mecanizados, aumentando el beneficio de los "grandes" y de las minorias locales poderosas y egoístas.

La descentralización de las decisiones en la construcción debe ir pues, unida a una descentralización tecnológica y administrativa, que abra paso a una tecnología adecuada de carácter más regional, que pueda ponerse en manos de: los usuarios (autogestión), las pequeñas empresas locales, cooperativas, comunas del interior, "consejos regionales", acción social, etc. para equilibrar la balanza y abrir nuevas perspectivas, imprescindibles e impostergables.

No es sólo cuestión de federalizar fondos, sino principalmente de definir y planificar una política habitacional, junto a una política científico-tecnológica, que enmarque y aliente nuevas propuestas para el hábitat, allí donde está la población y no desde la oficina ubicada en la ciudad lejana.

\section{TECNOLOGIAS ADECUADAS PARA PAISES EN DESARROLLO}

"Si las poblaciones pobres por cuenta propia y con tan pocos recursos consiguen tanto, ¿de qué no serian capaces si contaran con la colaboración y ayuda que reivindican?" (1)

Gandhi hizo surgir la "rueca" como símbolo de autoabastecimiento e independencia de la tecnología imperial, cuyo objetivo no era sólo satisfacer las necesidades esenciales de los pobres, sino quitarle fuerza al negocio monopólico y la dominación. Debemos inventar diversas "ruecas" en relación a vivienda progresiva, abastecimiento, transporte y almacenamiento de agua, energía simple local, nuevas formas de crédito, médicos y arquitectos de "pies descalzos", autoabastecimiento en la pequeña y productiva huerta familiar y grupal,

(1) "Tierra, Vivienda y ambientes pobres" - Conferencia Episcopal Brasileña. Revista del CIAS N. ${ }^{\circ} 314$ - Julio 1982. 
abonos naturales simples y a la mano de todos, sistemas de educación básica y lecto-escritura en relación a los reales problemas de la promoción y la justicia, saneamiento sanitario e higiene elemental realista y posible para todos, etc.

Esto no implica imponer formas atrasadas de tecnología para todos, ni de tener una tecnología para ricos y otra para pobres, sino de generar tecnologias locales y alternativas que apunten a cubrir prioritariamente y en forma creciente, las necesidades de las mayorias.

Claro está que una generalizada sobriedad y justicia debe primar desde el campo político y económico-social, para asegurar la coherencia interna de una sociedad, y destruir la superposición de dos mundos que se rozan, a veces en un mismo territorio: el mundo del desarrollo y la opulencia y el mundo mayoritario de los pobres y marginados.

"La persistencia de la injusticia, la falta de justicia amenaza la existencia de la sociedad desde adentron

"mientras persista un abismo profundo entre una minoria muy fuerte de ricos, por una parte, y una mayoria que viven en la necesidad y la miseria, por otra" (2)

En el mundo actual, injusto y conflictuado, donde predominan tecnologias incompatibles con la necesidad de cambio, el camino no puede hallarse en el creciente perfeccionamiento de la tecnología de avanzada, sino en el desarrollo de nuevas alternativas acordes con una nueva visión, más realista, plural y comunitaria.

Una red de información al respecto de objetivos, fines, procesos, aplicación de descubrimientos y entrenamiento de recursos en esta línea, está en movimiento, pero ciertamente aún es sumamente incipiente en relación a los poderosos medios con que cuenta la tecnología comercial y de avanzada.

\section{LA LUCHA POR UNA TECNOLOGIA DE VI- VIENDA ADECUADA PARA LAS MAYORIAS}

La adopción y el apoyo estatal a las tecnologías desarrolladas en los paises ricos, como exclusiva manera de resolver el déficit habitacional, no debe continuar cerrando el camino en los paises del hemisferio sur a la vivienda informal y el desenvolvimiento orgánico de tecnologias más apropiadas locales y zonales, según las reales posibilidades de satisfacer demandas masivas de vivienda básica y progresiva.

Por un lado el medio profesional y también la dirigencia política, productos de la uuniversidad de

(2) Juan Pablo II -Estadio Morumbi- Brasil, 3 de junio de 1980 Ediciones Paulinas. "Viaje a Brasil del Papa". Septiembre 1980 página 104. la Torre de Marfil", insiste generalmente con argumentos diversos, en tomar como única medida generalizable la óptica de los grandes centros mundiales.

Por otra parte, en la óptica del cambio, la tecnología adecuada está recién en sus orígenes. Pasada la primera euforia de la moda en los centros universitarios de Europa y del Tercer Mundo, es necesario continuar trabajando seriamente con el objetivo concreto de colaborar activamente en la solución del déficit habitacional, unida a la promoción de las masas.

La cuestión de las tecnologias habitacionales está condicionada ciertamente por la realidad socioeconómica, pero este condicionamiento no debe entenderse como un determinismo. Existe siempre un margen de posibilidad de cambio que debe utilizarse.

"Cuanto más estrictas son las limitaciones económicas, más necesarias son las iniciativas creadoras. Cuanto más alienante es el medio cultural, mayor debe ser la participación de la población en la producción y distribución de medios habitacionales."

"Cuanto más marcantes son las caracteristicas del medio ambiente natural, mayor es la urgencia de diseños concebidos para aprovechar esas condiciones naturales. Cuanto más difíciles son en suma, las condiciones del desarrollo, mayor es la necesidad de tecnologías apropiadas 0 adecuadas para la construcción del hábitat humano." (3)

El desarrollo de tecnología adecuada debe surgir como una respuesta realista a la satisfacción de necesidades masivas esenciales, recuperación cultural e incorporación progresiva de las masas a la vida moderna y la sociedad productiva, sanamente modificadas.

Esto a su vez debe comprender un uso más racional de los recursos locales, mano de obra abundante, materiales naturales y productos manufacturados de la zona, capitales, etc., buscando un resultado habitacional que concilie culturas locales aún vigentes, con el concepto de vivienda y hábitat contemporáneo. (4)

(3) "Taller de tecnologias apropiadas para un hábitat humano". Prospecto - Bogotá- Agosto 1983

(4) En esta linea por ejemplo la bloquera CINVA-RAM es una expresión de ingenio, economia y eficacia, tanto como el "baño por aspersión" (para economia de agua), los calefactores solares de fabricación casera el sistema Mas, el mueble sanitario y las ventanas de hormigón del CEVE, el Clivus Multrum de Suecia, etc.

Las diversas construcciones de adobe racionalizado y suelo cemento el sistema Simplex Cepol del Arq. Zacarelli en Chile (utilizado en las Fábricas Populares de Vivienda), los elementos premoldeados de Servivienda en Bogotá, los calentadores de biogas de la China Continental, el uso de la caña tacuara simplemente tratado en Africa y Filipinas, el uso de bloques de azufre del Arq. Ortega en paises del Medio Oriente, etc., ilustran brevemente la creatividad que puede volcarse en la construcción de la vivienda económica. 
Es necesario lograr productos económicos, de calidad, estabilidad, apariencia, resistencia, adición y reparación similares a los de la construcción tradicional, con amplias posibilidades de combinación y ductilidad a diversas propuestas arquitectónicas, de acuerdo a diseños y culturas locales.

Por otra parte esta visión requiere diseñar el proceso de construir de modo tal que posibilite la participación popular en el mismo y la apropiación de las tecnologias.

"Dale un pez, a un hombre necesitado y le ayudarás hoy, enséñale el arte de pescar y él se ayuadará a sí mismo." (5)

Pero "el arte de pescar" presupone a su vez facilitarle una caña y ésta (sobre todo si es de compleja factura), genera una nueva forma de dependencia técnica del proveedor; pero si se le enseña también a hacer su propio aparejo de pesca en forma simple, no sólo se le habrá ayudado a asegurar su autoabastecimiento, sino también a confiar más en si mismo y ser más independiente.

Asi por ejemplo, en lo tocante a vivienda es necesario: disminuir horas de trabajo, simplificar herramientas y procedimientos utilizables por una abundante mano de obra disponible, hacer más humana y libre cada tarea sin necesidad de un pesado aprendizaje tanto de la fabricación de partes como de la construcción o montaje, igualar las posibilidades constructivas de los que saben y los que no saben, permitiendo la incorporación de mujeres y aún niños.

Por último esta óptica implica dar cabida a que el proceso de construir pueda en muchos casos ser utilizado como medio aglutinante para la organización de comunidades en búsqueda de nuevos modelos de participación social (a través de Planes de Vivienda y Promoción) (6).

En éstos, las tecnologias apropiadas deben conformar "paquetes" de técnicas simples e integradas: constructivas, socio-organizativas, educativas, legales, financieras, etc. de posible apropiación y adecuación por parte de los grupos o comunidades que participen en el proceso organizativo y constructivo de la Ayuda Mutua, accediendo no sólo a la vivienda sino a la autogestión y siendo menos dependientes del poder del dinero y del poder politico.

En suma, el desarrollo de tecnología adecuada exige no sólo elaborar diversas propuestas técnicas, equipos y herramientas para los pobres, sino ( $y$ esto es lo más importante) conocer y trasmitir

(5) "Small is Beautiful" - E. F. Schumacher-Madrid, Ed. Blume año 1979 (O.C.), página 165.

(6) En este campo, entre los ejemplos más relevantes de lberoamérica se destacan los programas de la Fundación Salvadoreña de Desarrollo y Vivienda Minima (EI Salvador). el "cómo se hace" para viabilizar una fácil apropiación y participación, es decir una incorporación de la tecnología a la propia cultura que presupone su libre uso, mejoramiento, adaptación y aporte creativo. En los paises del tercer mundo exige también que facilite la participación de las mayorias marginales, organizadas en múltiples agrupaciones y comunidades que lleguen a la autogestión.

\section{VIVIENDA Y PROMOCION}

Esta idea de apropiación del "saber hacer" y "conocer cómo", cuando excede a los aspectos meramente constructivos y forma parte de un itinerario liberador, se asienta sobre la organización grupal y participativa, eje del proceso de construcción estrechamente vinculado a un proceso de promoción comunitaria. Esta concepción, de acuerdo a numerosas y variadas experiencias realizadas en países en desarrollo, nutre un amplio campo de posibilidades, del cual los Estados generalmente están ausentes.

Los prejuicios capitalistas hacen ver como "revolucionaria» toda forma de organización grupal con fines que apunten a toda acción solidaria o reivindicativa. A la inversa, para los sectores radicalizados de izquierda, todo lo que no sea repetir la receta del Partido, es presentado como "aspirina» paralizante (7).

La idea de una acción de "vivienda y promoción", pone el acento en el proceso de organizarse para cumplir el doble objetivo: construir la vivienda y desarrollar la autogestión comunitaria, apuntando a los fines de colaborar en un proceso de participación y desarrollo social.

Los planes para la auto-construcción de la vivienda grupal, alientan la construcción de la familia y nuevas formas de organización y vida comunitaria siendo servicios socio-habitacionales apoyados en el esfuerzo compartido, en el descubrimiento y desarrollo de capacidades, en la consolidación de grupos existentes o nuevos, en la interacción entre: usuarios, técnicos o agentes, sociedades intermedias y gobiernos.

Para los clásicos esquemas politicos, la vivienda conlleva sólo un problema financiero, ignorándose la importancia que puede tener su concreción, como signo y como aglutinante para la promoción y el cambio en los sectores de pobreza y de miseria que en la mayoría de nuestros paises son el grueso de la población.

Conviene destacar, por otra parte, que seria ingenuo pensar en términos de visualización de pro-

(7) Los sectores progresistas todo lo postergan a la realización de la "Revolución Salvadora", que todo lo hará bien por añadidura. Los sectores del "Statu quo" buscan mantener las cosas como están "deslizando algunas migajas" de las mesas de los ricos. 
32

Informes de la Construcción. Vol. 36, n. ${ }^{\circ} 362$. julio, 1984

gramas de vivienda (de mayor envergadura) en esta óptica, sin un apoyo creciente y más decidido de los propios gobiernos para ampliar experiencias y decidir nuevas políticas habitacionales y sociales.

La realización de uplanes de vivienda y promoción" apunta a los fines (8) de colaboración en la construcción de una sociedad más justa, libre y solidaria, lo cual significa:

Fines: 1. Un más justo reparto de los beneficios de la sociedad, entre los cuales está la vivienda.

2. Participación creciente de todos los sectores de la sociedad.

3. Desarrollo integral del hombre y la comunidad.

Objetivos: 1. Organización para la construcción de la vivienda propia y el barrio.

2. Crecimiento de las capacidades de los intervinientes en la apropiación de tecnologías adecuadas para Ilegar a la autogestión.

3. Ejercicio de una experiencia fructifera de "hacer juntos" tendiente a crear capacidad de asociación.

\section{PARTICIPACION Y "PAQUETES TECNOLOGICOS INTEGRALES"}

La tecnología adecuada, interdisciplinaria e integradora, correspondiente a este tipo de planes, toma a la vivienda progresiva no como un fin, sino como un medio eficaz de promoción y cambio.

Este camino indica una visión integrada del problema "vivienda y promoción", que necesita de la interdisciplina para construir la vivienda, el hábitat, la familia y la comunidad, con la participación libre y activa de todos los integrantes del grupo, y la de los agentes externos en una óptica de subsidiariedad.

Cada programa de "vivienda y promoción básica", debe presuponer una "acción múltiple» y unitaria y no la "adición" de varios proyectos paralelos.

(8) A menudo se confunden "Fines" y "objetivos" y éstos con expresiones de "deseo". Es un grave error para los técnicos confundir "expresiones de deseo" con objetivos y transmitir la confusión a los destinatarios de un programa, creándoles falsas expectativas e ilusiones Los objetivos nos "orientan" (buscar el oriente) concretamente hacia la acción. Se considera ubjetivon a todo aquello para 10 que en alguna medida se asignan recursos, trabajo, tiempo, etc. a los fines de lograr la transformación deseada. (Curso Dr. Francisco Suárez - AVE 1982). "Fin": motivo por el que se ejecuta una acción. (Enciclopedia Salvat).
Es decir, "la planificación del desarrollo grupal", a través de la realización de un "servicio habitacional», servicio que denote: diseño y ejecución, metodológicamente encarados de una serie de operaciones coordinadas, con el respaldo de este "paquete tecnológico adecuado" (constructivo, socioorganizativo, educacional, financiero, legal, etc.).

No es lo mismo sumar tareas de varias disciplinas que la puesta en obra de un plan integral.

Tampoco es igual utilizar tecnologias inconexas y propias de obreros calificados o de especialistas, que utilizar un conjunto integrado de tecnologías adecuadas para autoconstrucción.

El motor de esta tarea mancomunada de: entes financieros, técnicos y usuarios, está constituido por el descubrimiento de las necesidades sentidas y comunes a todo el grupo destinatario y el desenvolvimiento de las capacidades personales y de organización comunitaria a través de la construcción de la vivienda.

Para desarrollar los usuarios su parte creativa, no sólo en la tarea física (construcción), sino en el saber asumir progresivamente la "planificación" (diseño y puesta en marcha del plan), se necesitan dos herramientas interrelacionadas: tecnologias adecuadas (paquete) y técnicos y promotores idóneos.

El objetivo integrado: "organizarse y construir la vivienda y el barrion de acuerdo a los fines explicitados, es a la vez el fundamento y signo sobre el que se apoya toda la tarea promocional.

Si el programa se dirige sólo a la materialización de la vivienda, puede suceder que poco se avance en aras de un verdadero camino de promoción, aunque sea indudablemente beneficioso en orden a la vida personal y familiar.

Pero también si la vivienda ha sido ejecutada deficientemente o de manera agobiante y sin el uso de una tecnologia constructiva adecuada, aunque el trabajo social complementario, desde la óptica de la propia técnica, haya sido correcto, estará asentado sobre una base endeble. La frustración en el logro del primer objetivo aglutinante: vivienda.

La idea de un servicio habitacional de promoción o vivienda de promoción, no se refiere a la construcción de viviendas con tecnologias convencionales y apoyo social para su mejor materialización, ni tampoco a la realización de programas promocionales que toman la ejecución de la vivienda exclusivamente como un trampolín para cumplir con determinados objetivos sociales y políticos.

No se trata aquí de definir teóricamente qué es más importante, si la posesión de una vivienda o 
dar un paso promocional con el pretexto de la vivienda. ¿Qué priorizar? ¿La vivienda que apunte a la estabilidad y promoción de la familia o el proceso organizativo que apunta a la solidaridad y la participación social? ¿El huevo o la gallina?

Dada la trascendencia que la organización grupal puede tener a partir de la experiencia de construir la vivienda y el hábitat propio, como también el arraigo, autodeterminación y valoración que generan la posesión y uso de ésta, debemos rescatar el objetivo central en este tipo de planes:

Organización para la construcción de la vivienda y el barrio como camino de afirmación familiar y autogestión comunitaria.

Para visualizar el complejo y a la vez sintético proceso de "vivienda y promoción" se presenta el siguiente gráfico:

\begin{tabular}{l}
\hline Capacitación \\
\hline Organización participativa de tecnologias \\
\hline Reflexión qruoal Participacion consciente \\
\begin{tabular}{|l|}
\hline Confort básico \\
\hline Uso de la vivienda propia \\
\hline Autoestima \\
\hline
\end{tabular} \\
\hline
\end{tabular}

Herramientas:

$\star$ paquete tecnológico

$\star$ técnicos 0 agentes subsidiarios

Sujeto del Proceso:

* grupo o comunidad con participación creciente.

En estos programas la "Ayuda Mutua" es el vehiculo de las tecnologías apropiables y por ello visualiza los objetivos comunitarios que apuntan al "proceso" y al "resultado: vivienda».

En lo que respecta al proceso, la mayor utilidad de la Ayuda Mutua uradica en las posibilidades educativas que propende como interacción social". (9)

Los planes de "vivienda y promoción" o "servicio habitacional integral» exigen acciones sintesis que implican, desde la gestación de las tecnologías concurrentes, una convergencia y simplificación efectivas, de manera tal que el desenvolvimiento de una se apoya en el desenvolvimiento de las demás, en función de requerimientos concretos.

Estos paquetes de tecnologias adecuadas para servicios habitacionales de promoción, requieren investigar, experimentar y desarrollar "caminos comprensibles y apropiables", intentando no sólo

(9) "El Hilo Conductor" - FSDVM - El Salvador, septiembre 1980, página 12. (Publicación guia que presupone una tecnologia educativa en relación a programas de vivienda por Ayuda Mutua y Esfuerzo Propion. reducir desajustes entre las diversas técnicas intervinientes, sino buscar su potenciación a través de su interrelación y mutuo apoyo.

La investigación, experimentación y evaluación ciclicas, intimamente ligadas, deben afirmar y ampliar estas experiencias para que lleguen a tomar progresivamente un lugar al lado de las tradicionales politicas públicas.

En tanto el medio sea todavia hostil e indiferente al desarrollo de tecnologias adecuadas, la insistencia en la realización de microproyectos y modelos experimentales debe ser más fuerte y persistente, como ya se hace en numerosos centros del Tercer Mundo, a veces con el apoyo de algunas instituciones y técnicos del Norte entusiasmados con estas herramientas de cambio.

\section{UNA EXPERIENCIA ARGENTINA}

La Asociación de Vivienda Económica (AVE) de Córdoba, Argentina, desarrolla como tarea central con sus dos centros: Investigación (CEVE) y Programas demostrativos de Servicio Socio-habitacional (SEHAS), la investigación e implementación de paquetes de tecnologías integradas, adecuadas para procesos socio-habitacionales de organización y participación comunitaria de sectores de escasos recursos en la solución de los problemas relacionados al hábitat.

El proyecto de Yapeyú que se describirá a continuación es uno de los primeros programas demostrativos en esta línea. En la misma orientación algunos de los más recientes programas y estudios, son:

"Plan de Vivienda. Desarrollo Comunitario. Fuente de Trabajo" (Pequeña empresa constructora) Chaco Chico (108 familias) -Córdoba- Argentina. Sistema constructivo MAS, CEVE, apoyo CEBEMO (Holanda).

«Plan Desarrollo Comunitario, Vivienda progresiva y participación en el Diseño" La Cortada - Santa $\mathrm{Fe}$ - Argentina (300 familias). Sistema constructivo MAS-CEVE. Apoyo: CARITAS. INCUPO. CEBEMO. FUNDAPAZ.

"Lotes, Servicios y Vivienda Progresiva y DesarroIlo Comunitarion. El Rosedal, Córdoba (119 familias). Estudios de procesos y evaluación de resultados. Financiación: CEBEMO.

"Pre-fachada, Vivienda progresiva y Participación Comunitaria" Plan Las Flores, Córdoba (11 familias). Financiación: F.I.A.

"Desarrollo Comunitario, Servicio de Agua, Vivienda, Fuente de Trabajo en estudio" (Granja comunitaria) La Merced, Córdoba (100 familias). Financiación: M.A.S. - MISEREOR. 
Investigación metodológica interdisciplinaria y upaquetes" de tecnologia adecuada. CEVE-SEHAS, con apoyo de FIA 1983-84.

Estudio y Propuesta sobre "Participación del usuario en el Diseño" (Area de Evaluación CEVE y Alumnos de la UNC y UCC. Primer premio UNESCO 1984. Concurso Internacional organizado por la U.I.A.

\section{UN EJEMPLO PILOTO: BARRIO ALTOS DE YAPEYU}

Se expone a continuación el resumen de una experiencia, ubicada como una de las pioneras realizadas en la Institución en la temática descrita. Se trata de un Plan Piloto donde se requeria investigar un modelo de construcción de viviendas creado en el Instituto y en la perspectiva de impulsar simultáneamente un proceso socio-organizativo, educativo y de fuente de trabajo en una comunidad.

Villas Altos de Yapeyú es un asentamiento de 29 familias asentadas desde varios años atrás en terrenos fiscales y con vivienda provisoria en la ciudad de Córdoba, Argentina.

El grupo humano vivia inicialmente en las márgenes del Río Primero y debido a las inundaciones producidas en el año 1972 habian sido trasladados por el Gobierno Provincial a esos terrenos.

Hasta el año 1977 no se habia modificado en nada esta situación, debiendo "los villeros" (de "villas miserian) soportar el rechazo de los vecinos de los barrios circundantes a este asentamiento. Si bien los miembros de la villa se encontraban en viviendas precarias, su nivel socio-económico era superior al de otros grupos similares, alcanzando el núcleo familiar un ingreso promedio de alrededor de US $\$ 100$ mensuales. Prácticamente todos los jefes de familia tenian trabajo (aunque no estable). El $100 \%$ de los niños en edad escolar concurría a la escuela pública. El porcentaje de delincuencia y alcoholismo era pequeño. Los grupos familiares (de 5 personas promedio) estaban nucleados en viviendas provisorias a fondo de lote. La edad promedio de los adultos era de 38 años.

A mediados de 1977, AVE-CEVE obtuvo recursos de la O.E.A. (dentro de su Programa Especial de Investigación de Vivienda Popular) para la ejecución de un Plan Piloto Experimental de viviendas por autoconstrucción. En esta situación se propuso a la comunidad la ejecución del mismo y se obtuvo la autorización del Gobierno Provincial para su ejecución.

En forma muy sintética, los objetivos propuestos, el desarrollo de la experiencia y las conclusiones del Programa, son:
OBJETIVO N. ${ }^{\circ}$ 1. Ensayar en una experiencia insertada en una comunidad concreta, una metodologia de asistencia técnica interdisciplinaria y un paquete tecnológico integral, a partir de la utilización del sistema constructivo MAS (diseñado para autoconstrucción). (Fig. 1).

DESARROLLO: En el proceso se utilizó un método de asistencia técnica que integraba los aspectos de construcción con los sociales y organizativos. Trabajaron para ello un equipo (arquitecto y asistente social) coordinado por el Instituto, y que apuntaba a una tarea interdisciplinaria de apoyo y estímulo al trabajo originado en la vivienda.

De común acuerdo con todas las familias del Plan se trazó un orden de ejecución de tareas. Cada paso a ejecutarse, además de su explicación previa, estuvo apoyado por un manual didáctico que podia ser consultado en cualquier momento. De esta manera se fue logrando una capacitación paralela al desarrollo del Plan. La función de los técnicos fue también apoyar mediante la organización para la construcción de estas viviendas el inicio de un diálogo reflexivo y análisis de posibilidades para que toda la comunidad se apropiara del sentido e importancia de desarrollar actividades que le permitiera mejorar el entorno natural físico y progresar como persona y grupo, con vistas a un proceso creciente de autogestión comunitaria.

CONCLUSION: Se comprobó una gran facilidad para el aprendizaje del sistema por parte del usuario (mano de obra no calificada). Trabajaron hombres, mujeres y niños con entusiasmo y eficiencia. El sistema admitió y toleró cómodamente las imprecisiones de esta mano de obra sin desvirtuarse técnicamente. Puede decirse que el modo de asistencia técnica interdisciplinaria empleado en el Plan es adecuado para ejecutar un proceso de vivienda y promoción en comunidades de estas caracteristicas.

OBJETIVO N. ${ }^{\circ}$ 2. Crear un modelo de espacio urbano acabado, que permita concretar una alternativa de interés frente al caos del crecimiento espontáneo o la geometría implacable del loteo, capaz de generar un ejemplo multiplicador y expresando una comunidad viviente.

Para ello se introduce "la prefachada" o "plano virtual ordenador", para delimitar el espacio comunitario.

Proponer una célula básica de vivienda minima, crecible con los servicios elementales.

DESARROLLO: Se buscó y localizó el Plan en el sitio adecuado y con un diseño que diera imagen de un conjunto urbano, modesto pero de calidad espacial, tendiente a ensayar un "Hábitat" característico, ordenado y a una escala apropiada para el sector. Se ejecutó el grupo de viviendas, la calle 


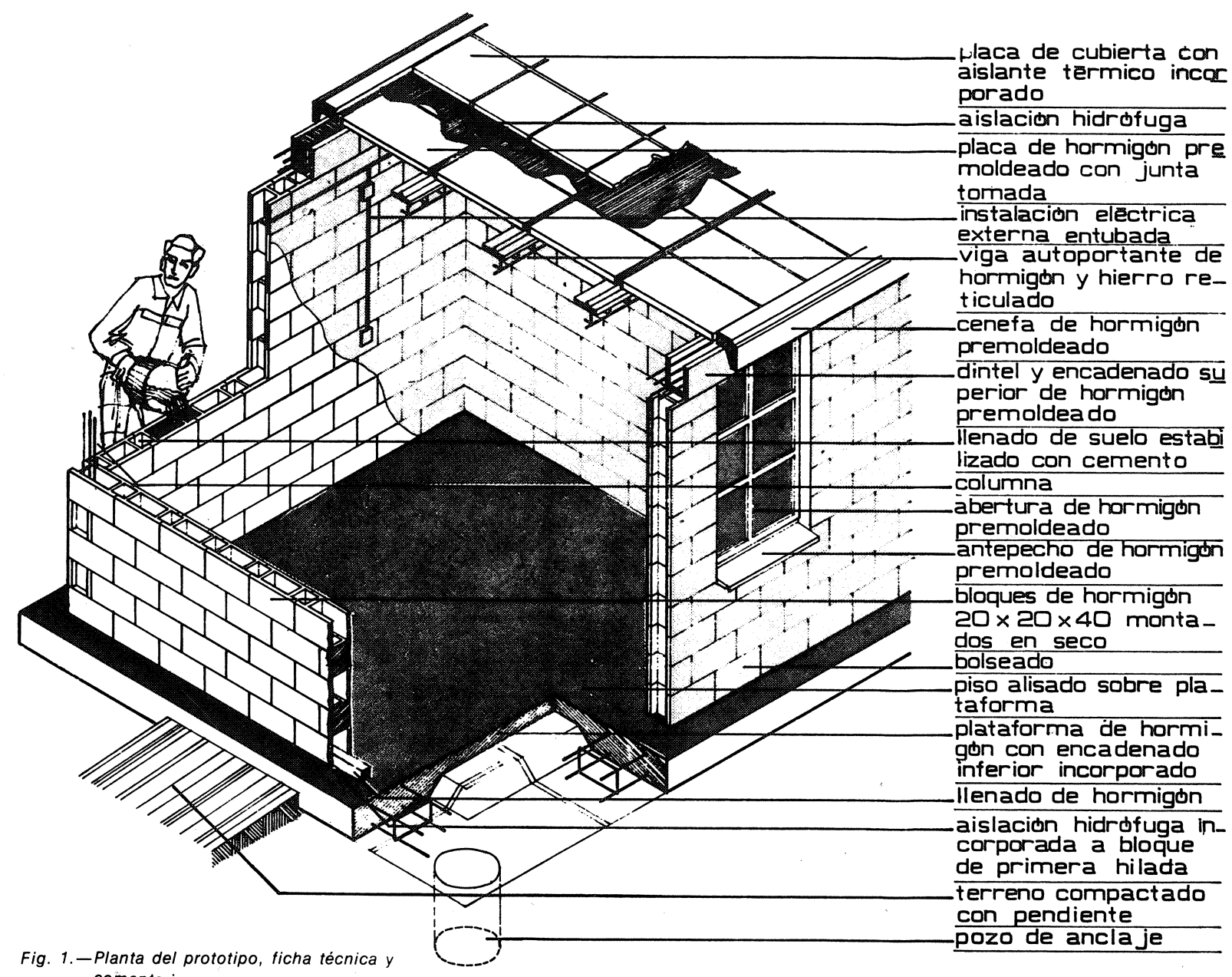
comentario.

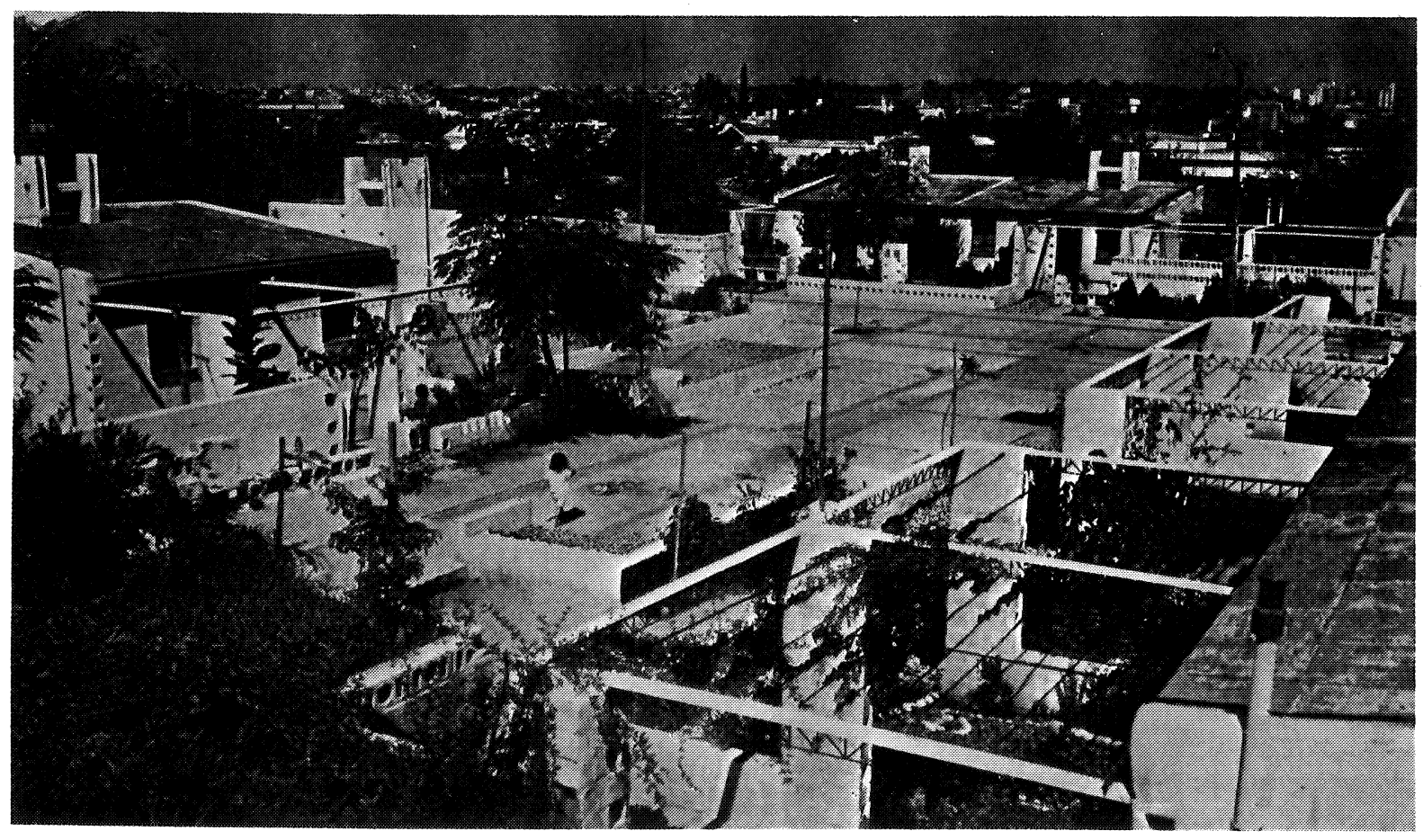

Fig. 2. - Vista desde un patio hacia la calle. 


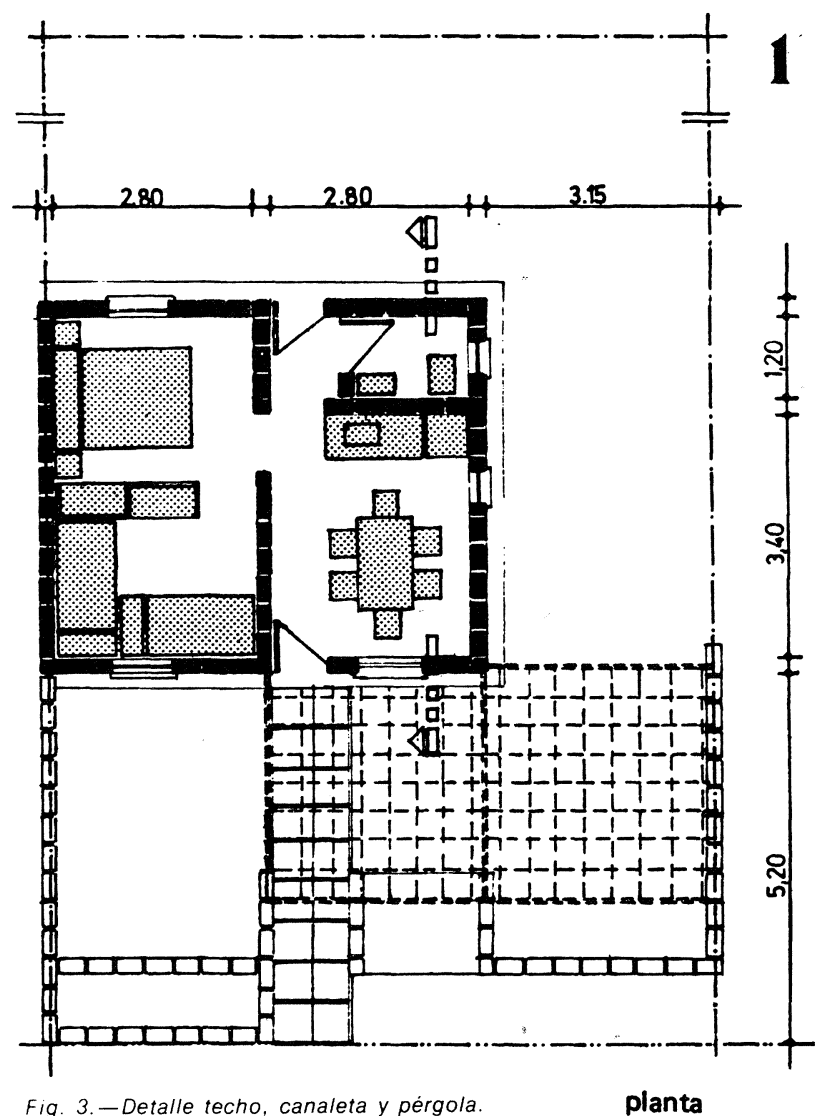

correspondiente (corazón del barrio) con veredas y arbolado, y un espacio común para uso de todas las familias. La financiación de esta mínima infraestructura urbana la hizo el Ministerio de Bienestar Social de la Provincia, y su administración estuvo a cargo de SERVIPROH (Servicio de Vivienda y Promoción Humana, asociación civil sin fines de lucro) entidad que trabajó conjuntamente con el CEVE. (Fig. 2).

Se diseñó una vivienda de $32 \mathrm{~m} 2$ que consta de cocina, baño completo y un dormitorio grande, con instalaciones eléctricas y aberturas completas. La unidad minima fue construida rápidamente y sin exagerados esfuerzos. Las familias trabajaron los sábados durante 10 meses. (Fig. 3).

CONCLUSION: La materialización de un rincón urbano demostrativo a través de un "soporte básico" del orden común (PREFACHADAS), resultó de gran simplicidad y riqueza espacial, abriéndose nuevos caminos al diseño participativo. La unidad habitacional básica, satisface necesidades mínimas y ha sido el inicio de un proceso de ahorro, canalizado al completamiento de la vivienda y equipo, demostrativo de un ascenso personal, familiar y grupal creciente y la debida apropiación de la tecnologia. Los usuarios han asumido lenta y firmemente su nuevo hogar y las nuevas responsabilidades adquiridas.

OBJETIVO N. ${ }^{\circ}$ 3. Desarrollar el Plan Piloto en un

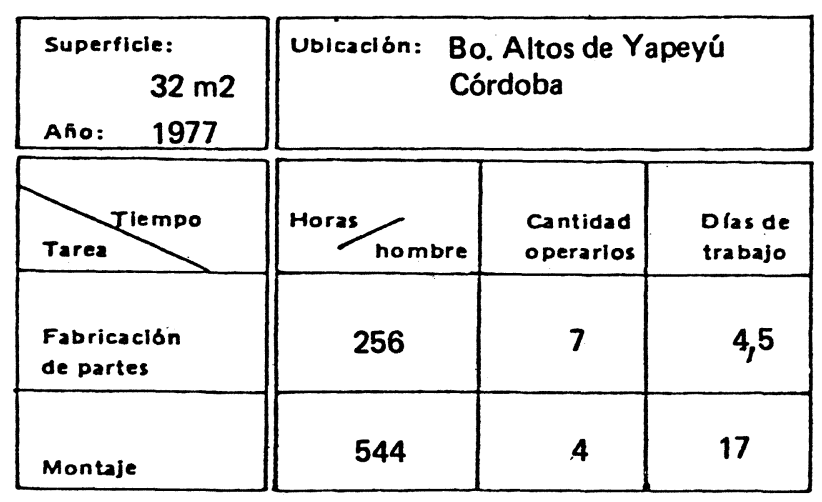

Prototipo de unidad mínima: un solo dormitorio divisible. Crecimiento hacia atrás por adosamiento o por pasillo de vinculación al fondo del lote.

El techo cambia levemente llevando la pendiente hacia adelante. El diseño está condicionado, como todos los presentados, por razones económicas.

En este caso, al tratarse de un plan, se aparean las casas de a dos para presentar una fachada más grande, lo que favorece al aspecto general.

Se consolida además la idea "prefachada".

Esta, se realiza mediante el uso de canteros, verjas y pergolado que conforman y caracterizan el espacio exterior. El uso de la galería verde caduca, como extensión del espacio en la vivienda de bajo costo, incorpora la naturaleza como participante activa de la conformación del espacio.

grupo marginal con un mínimo de organización y capacitación previa para evaluar el alcalce de la promoción lograda a través de la construcción de la propia vivienda y un programa básico de promoción social y organización participativa.

DESARROLLO: La comunidad marginal carecia de organización social previa. Fue necesario promover la formación de un grupo representativo del barrio a fin de faci!itar algunas de las tareas concretas del Plan y estimular la necesidad de crear y mantener su entorno físico propio y de conjunto, e incrementar la participación como primer paso para la promoción humana, para que cada uno se sintiera protagonista de realizaciones que no sólo los beneficiara individualmente sino a todos.

CONCLUSIONES: Se obtuvo una estructura básica de organización social a nivel de comunidad en general y a nivel de las familias del Plan Piloto, por el "Esfuerzo Propio" y "Ayuda Mutua" convenientemente desarrollados.

La comunidad comprobó la posibilidad real de ejecución de un plan de estas características, adquiriendo así un grado de confianza en sus propias capacidades. Individualmente este Plan permitió ejercitar la capacidad de "hacer" participando activamente en la solución de una necesidad propia a través de lo cual les fue posible descubrirse como seres capaces, lograr autoafirmación y reconocimiento de valores y condiciones propias. 
OBJETIVO N. ${ }^{\circ} 4$. Experimentar en pequeña escala la creación de un "circuito" de producción y consumo, donde actúe el equipo técnico como grupo de Coordinación y Asistencia Técnica de tres comunidades marginales organizadas como Cooperativas de Trabajo que proveen algunos de los componentes de construcción utilizados, desarrollados con tecnología del CEVE.

DESARROLLO: Además del montaje de elementos constructivos por autoconstrucción, el programa organizó una red abastecedora (fabricantes) de estos mismos elementos con la concurrencia de otras comunidades marginales, que, constituidas en Cooperativas de Trabajo, estaban en condiciones de fabricar y vender componentes. El hecho de que la tecnología del sistema era sencilla y no requeria de herramientas o equipos especiales, no sólo abarató los costos y dio trabajo a dichas Cooperativas, sino que probó y facilitó la posibilidad de auto-fabricación de partes coordinadas.

La Cooperativa "20 de junio" fabricó las ventanas de hormigón y losetas. Por su parte la Cooperativa "San José" hizo lo propio con las viguetas del techo, mientras que la Cooperativa "25 de Mayo" realizó todo el trabajo "in situ" de veredas, calles, escaleras, canteros. En el mismo barrio se hicieron, en cambio, todos los bloques y dinteles premoldeados.

CONCLUSIONES: La obra fue correctamente abastecida por sus proveedores, en los costos y términos precisos, reforzándose la idea de interacción cooperativa en un circuito de economia local, autogestión y promoción de la pequeña industria.

La experiencia descrita en resumen aquí, se halla, publicada bajo el título "Vivienda y Promoción Humana: Un programa piloto" (120 páginas - Ediciones AVE 1979). Autores L. Rivarola y A. Ferrero.

\section{CONCLUSION}

Finalizando, se puede decir que para contribuir positivamente a la solución del problema de la vivienda en Iberoamérica y en general en el Tercer Mundo, es necesario tener en cuenta las profundas intenciones tecnológicas que definen a grandes rasgos dos grupos de alternativas complementarias.

La tecnología de la vivienda llave en mano que avanza aceleradamente por el camino de interrelacionar tareas mecanizadas de creciente especialización, que se ajusten a esquemas programados, precisos; y por otro lado la tecnología constructiva adecuada para la autoconstrucción que avanza lentamente en la búsqueda de lograr tareas simples, humanizadas y creativas, de fácil aprendizaje y formas de aplicación comunitaria, que permitan la participación y el intercambio de roles entre los participantes a través de un dúctil esquema organizativo.

La primera mira más al producto terminado y su creciente perfección y confort para adecuarse a una cada vez más refinada competencia de mercado.

La segunda apunta tanto al producto "vivienda progresiva" como al procedimiento constructivo, núcleo del proceso de organización participativa para la autogestión.

De aquí la importancia que tiene la tecnologia constructiva como elemento aglutinante de las demás y no sólo como vehículo para materializar una vivienda.

Repetimos que la vivienda convencional para las masas (fuera de los paises ricos del Norte), es simplemente la expresión de un sueño hermoso sometido al "acaso" de un gran despegue económico, o de una revolución salvadora.

No podemos esperar riquezas fáciles, ni revoluciones salvadoras... la única riqueza y la única salvación está en la fuerza y el corazón de los propios hombres organizados para solucionar sus problemas y a la vez generar las presiones y reivindicaciones de justicia, necesarias para obtener del poder político el debido apoyo.

Por eso, junto a las tecnologias convencionales para la "vivienda llave en mano", usadas por las compañias constructoras privadas o del Estado, comprobamos la urgencia de desarrollar tecnologías adecuadas o apropiadas para satisfacer necesidades elementales de las distintas regiones o paises en desarrollo.

El saber académico y formal toma a la tecnologia convencional, generalmente determinada y producida en los grandes centros del poder mundial, como la única válida, y no suele tener conciencia de la importancia de desarrollar respuestas más realistas y nuevas alternativas acordes con las necesidades y posibilidades de las mayorias.

Todas las culturas supieron crear su tecnología adecuada, no sólo para dar respuesta a sus necesidades materiales, sino también espirituales. EI producto final útil, bello, simple y económico, era tan importante como el proceso: creativo comunitario, en relación con el medio natural, la transmisión familiar y sobre todo la relación con una economía de subsistencia básica de las masas.

Mejor tecnologia, más simple y económica, apta para la "autoconstrucción", la "ayuda Mutua", "las microbrigadas", "sistemas mixtos", "compañias comunitarias" o "cooperativas de vivienda", etc. es el apoyo mínimo que necesitan nuestros pueblos para dar respuesta, urgente y masivamente a su problema habitacional y social. 